\title{
Publisher Correction: An engineered ScCas9 with broad PAM range and high specificity and activity
}

Pranam Chatterjee (D), Noah Jakimo, Jooyoung Lee (D), Nadia Amrani (D), Tomás Rodríguez, Sabrina R. T. Koseki (1), Emma Tysinger, Rui Qing (i), Shilei Hao (1), Erik J. Sontheimer (i) and Joseph Jacobson

Correction to: Nature Biotechnology https://doi.org/10.1038/s41587-020-0517-0, published online 11 May 2020.

In the version of this article initially published online, $\mathrm{Sc}^{++}$was given as $\mathrm{Sc}^{+++}$in two places in the fifth paragraph of the Results section. The error has been corrected in the print, PDF and HTML versions of the article.

Published online: 19 June 2020

https://doi.org/10.1038/s41587-020-0604-2

(c) The Author(s), under exclusive licence to Springer Nature America, Inc. 2020

\section{Publisher Correction: The making of the Biohub}

Laura DeFrancesco

Correction to: Nature Biotechnology https://doi.org/10.1038/s41587-020-0685-y, published online 11 September 2020.

In the version of this article initially published online, the Biohub's full name was given in two locations as CZI Biohub; the correct name is Chan Zuckerberg Biohub. The last name of California's governor, Gavin Newsom, was given as Newsome, and the first name of the Biohub's COO, Gajus Worthington, was given as Gaius. Amy Kistler was identified as an epidemiologist; she is infectious disease group leader. CZI was described as a not-for-profit; it is now described as a philanthropy. Finally, the relationship among entities was mischaracterized: "The pivot on a dime was possible because DeRisi is part of the Chan Zuckerberg Initiative" has been changed to "The pivot on a dime was possible because the Biohub is funded by the Chan Zuckerberg Initiative." The errors have been corrected in the print, PDF and HTML versions of the article.

Published online: 17 September 2020

https://doi.org/10.1038/s41587-020-0702-1

(C) Springer Nature America, Inc. 2020 